\title{
Criança hospitalizada: caracterização dos procedimentos cirúrgicos em um hospital escola público
}

\section{Hospitalized child: characterization of surgical procedures in a public school hospital}

\author{
Barbara de Andrade Alves'; Tabatha de Freitas Moreira Santos²; Rosângela Aparecida \\ Pimenta Ferrari ${ }^{3}$; Mauren Teresa Grubisich Mendes Tacla ${ }^{4}$; Flávia Lopes Sant'Anna; \\ Emilia Batista Lopes ${ }^{6}$
}

\begin{abstract}
Resumo
Este trabalho teve como objetivo caracterizar os procedimentos cirúrgicos realizados em crianças internadas na unidade pediátrica de um hospital escola público. Trata-se de pesquisa quantitativa, descritiva e transversal, realizada em Londrina, Paraná, no período de janeiro de 2007 a dezembro de 2009. Utilizou-se como fonte de dados o sistema de cadastro do serviço de estatística do hospital, o qual forneceu prontuários para análise. Durante os três anos de estudo ocorreram 2.360 procedimentos, dos quais $56,7 \%$ foram em crianças provenientes do próprio município. Houve predomínio do sexo masculino $(62,0 \%)$ e da faixa etária de um a três anos $(30,0 \%)$. A clínica responsável pelo maior número de procedimentos foi a cirurgia infantil $(54,6 \%)$, seguida pela ortopedia $(14,8 \%)$ e hematologia $(12,1 \%)$. Os procedimentos mais realizados foram apendicectomia, punção liquórica e correção de fraturas. Correção de malformações congênitas totalizaram $10,8 \%$, e procedimentos decorrentes de causas externas $16,0 \%$. Ocorreu apenas um óbito no intraoperatório nos três anos de estudo. Os resultados permitiram caracterizar o perfil das cirurgias realizadas nesta unidade e identificar o aprimoramento de técnicas específicas de manejo e preparo pré e pós-operatório para melhor assistência à criança e família, com redução de possíveis traumas decorrentes da hospitalização.
\end{abstract}

Palavras-chave: Cirurgia. Pediatria. Cuidado da criança. Enfermagem pediátrica.

\begin{abstract}
This study aimed to characterize the surgical procedures performed in children hospitalized in the pediatric unit of a public school hospital. It is a quantitative, descriptive and cross-sectional study conducted in the city of Londrina, Paraná, from January 2007 to December 2009. Data were collected from the hospital's statistical department database record, assessing medical records of patients. During the three years of study, 2360 procedures were performed, of these $56.7 \%$ were in children who lived in the city itself. There were predominance of males $(62.0 \%)$ and age group between 1 and 3 years old $(30.0 \%)$. The larger amount of
\end{abstract}

\footnotetext{
${ }^{1}$ Enfermeira. Mestre em Enfermagem, Departamento de Enfermagem, Centro de Ciências da Saúde, Universidade Estadual de Londrina.

${ }^{2}$ Enfermeira. Especialista em Saúde da Criança. Departamento de Enfermagem, Centro de Ciências da Saúde, Universidade Estadual de Londrina.

${ }^{3}$ Enfermeira. Doutora em Ciências. Professor Adjunto e Coordenadora da Residência em Enfermagem em Saúde da Criança, Departamento de Enfermagem, Centro de Ciências da Saúde, Universidade Estadual de Londrina.

${ }^{4}$ Enfermeira. Doutora em Enfermagem. Professor Adjunto e Vice-coordenadora da Residência em Enfermagem em Saúde da Criança, Departamento de Enfermagem, Centro de Ciências da Saúde, Universidade Estadual de Londrina.

${ }^{5}$ Enfermeira. Doutoranda em Saúde Coletiva. Professor Auxiliar, Departamento de Enfermagem, Centro de Ciências da Saúde, Universidade Estadual de Londrina.

${ }^{6}$ Enfermeira Residente de Enfermagem em Saúde da Criança, Departamento de Enfermagem, Centro de Ciências da Saúde, Universidade Estadual de Londrina.
} 
procedures was performed by the pediatric surgery clinic (54.6\%), followed by orthopedics $(14.8 \%)$ and hematology (12.1\%). The most frequent procedures were appendectomy, liquor puncture and orthopedic fracture correction. Congenital malformations correction amounted $10.8 \%$ and procedures resulting from external causes $16 \%$. Only one death occurred during the intraoperative period in three years of study. The results allowed to characterize the performed surgeries' profile, providing subsidies for improving specific techniques for management and pre-and postoperative preparation to ensure better care for child and family and reduction of possible traumas during hospitalization.

Keywords: Surgery. Pediatrics. Child Care. Pediatric Nursing.

\section{Introdução}

Para a maioria da população a hospitalização é uma situação perturbadora. Pior ainda para as crianças, uma vez que atinge um grau ainda maior, podendo acarretar em diversos traumas. A internação, para este grupo etário, é um acontecimento estressante, pois o afasta da rotina familiar e o insere em meio desconhecido, onde sua segurança é frequentemente ameaçada. Somase a este fato, a incapacidade da maioria delas compreender a natureza de tais acontecimentos (QUIRINO; COLLET, NEVES, 2010).

Além dos desconfortos causados pela internação, alguns autores referem ainda que quando esta se motiva por procedimento cirúrgico, a criança pode vir a desenvolver distúrbios psicológicos, advindos da ansiedade e das situações com as quais não está acostumada, como ter contato com pessoas desconhecidas e sentir dor. Tudo isso pode contribuir e intensificar reações de medo e insegurança (BROERING; CREPALDI, 2011).

O paciente pediátrico possui peculiaridades que devem ser avaliadas quanto aos aspectos anatômico, fisiológico e resposta às drogas utilizadas, havendo a necessidade de interação entre pediatra, anestesiologista, cirurgião, criança e pais (CARAVALHO et al., 2010; STEIN, 2006). Além desses profissionais, o enfermeiro, fisioterapeuta, psicólogo, assistente social e terapeuta ocupacional também precisam avaliar aspectos sociais e psicológicos inerentes à hospitalização da criança para amenização do sofrimento infantil (SOARES; LEVENTHAL, 2008). Informações como idade, nível de desenvolvimento, compreensão da criança e da família sobre sua condição física, tipo de procedimento a ser realizado, experiência prévia de hospitalização e medo de procedimento específico auxiliam neste processo, bem como permitem traçar método mais apropriado e individualizado para preparo pré, trans e pós-operatório (BROERING; CREPALDI, 2011).

É de fundamental importância a participação dos pais durante a hospitalização e no planejamento da alta, pois eles representam para criança maior segurança, tornando-a mais colaborativa no tratamento, acelerando o processo de recuperação e amenizando a internação traumática (SOARES; LEVENTHAL, 2008). À medida que os familiares são integrados no cuidado há maiores possibilidades no trato da criança, pois se incorporam peculiaridades individuais tornando a sistematização da assistência eficaz e eficiente.

Entretanto, para que ocorra abordagem individualizada, o serviço de saúde deve ofertar recursos humanos e de infraestrutura qualificados para realização de procedimento cirúrgico eletivo ou de urgência/emergência. Cada situação exige rotinas diferenciadas (BROERING; CREPALDI, 2011; CORONEL, 2011; STEIN, 2006).

Na situação de urgência/emergência o sofrimento é intenso e inesperado, tornando o ato cirúrgico condição que afetará a integridade física da criança, embora seja a única indicação para sua recuperação. A família estará mais fragilizada emocionalmente, e precisará subitamente se organizar para permanecer no hospital. A atuação da equipe multiprofissional 
será a de oferecer apoio e orientações pertinentes ao grau de comprometimento do agravo, com seguimento na sistematização da assistência no pós-operatório (BROERING; CREPALDI, 2008; BROERING; CREPALDI, 2011; CARAVALHO et al., 2010; CORONEL, 2011; QUIRINO; COLLET; NEVES, 2010; SOARES; LEVENTHAL, 2008; STEIN, 2006).

Visto que as situações de hospitalização infantil podem ser diversificadas, a caracterização da população pediátrica cirúrgica admitida em ambiente hospitalar pode melhorar o direcionamento assistencial, assim como a adequada atuação multiprofissional à criança e sua família. Dessa forma, o presente estudo teve como objetivo caracterizar os procedimentos cirúrgicos realizados em crianças internadas na unidade pediátrica de um hospital escola público do sul do Brasil.

\section{Método}

Trata-se de um estudo quantitativo, descritivo e transversal, realizado em um hospital escola público do município de Londrina, Paraná, no período de 01 de janeiro de 2007 a 31 de dezembro de 2009 .

O hospital de estudo é um órgão público universitário vinculado à Secretaria de Estado da Ciência, Tecnologia e Ensino Superior. Trata-se de centro de referência regional para o Sistema Único de Saúde (SUS), considerado único hospital público de grande porte do Norte do Estado do Paraná. A unidade pediátrica é constituída por 34 leitos e recebe crianças entre zero e 12 anos incompletos de diversas especialidades, com média mensal e anual de 49 e 580 internações, respectivamente.

A casuística foi composta pelo cadastro de 2.360 procedimentos cirúrgicos realizados em crianças nesses três anos de estudo. Uma mesma criança pode ter sido submetida a mais de um procedimento cirúrgico nesse período.

Os dados foram disponibilizados pelo sistema de cadastro do serviço de estatística do hospital, no qual foi possível identificar: nome da criança, idade, sexo, tipo de cirurgia, clínica responsável e data do procedimento cirúrgico realizado. Esses dados foram transcritos em formulário específico e processados eletronicamente pelo programa Microsoft Excel®. Posteriormente foram organizados em tabelas e figuras.

A pesquisa seguiu todas as normas referentes à Resolução n. 196/96 e recebeu aprovação do Comitê de Ética em Pesquisa e autorização da instituição pelo parecer no. 236/08, CAAE no. 0233.0.268.000-08.

\section{Resultados}

Nos três anos de estudo 6.518 crianças foram internadas na unidade pediátrica do hospital, média de 181 internações ao mês. Destas, 1.292 (19,8\%) foram submetidas a procedimento cirúrgico. Houve casos em que uma mesma criança sofreu mais de uma intervenção, resultando em casuística final de 2.360 procedimentos.

Em relação à procedência da criança, predominou a cidade de Londrina (56,7\%). Outros municípios de abrangência da $17^{\mathrm{a}}$ Regional de Saúde de Londrina totalizaram 23,5\%, 18,6\% eram de outras regionais do Paraná, e 1,2\% correspondeu aos Estados de São Paulo, Mato Grosso do Sul e Pará.

A Tabela 1 mostra os procedimentos cirúrgicos de acordo com a idade e a clínica responsável pela internação da criança. A maioria compreendeu a faixa etária de um a três anos $(30,0 \%)$ e de sete a nove anos $(23,5 \%)$. Com relação à clínica, predominou a cirurgia infantil $(54,6 \%)$, seguida pela ortopedia $(14,8 \%)$ e hematologia $(12,1 \%)$. Os demais procedimentos foram realizados pela bucomaxilo, neurocirurgia, odontologia, oftalmologia, otorrinolaringologia e palatoplastia. 
Tabela 1- Procedimentos cirúrgicos por clínicas e faixa etária das crianças internadas na unidade pediátrica de um hospital escola público, 2007-2009, Londrina (PR).

\begin{tabular}{|c|c|c|c|c|c|c|c|c|c|c|}
\hline \multirow{3}{*}{$\begin{array}{l}\text { Faixa } \\
\text { Etária } \\
\text { (em } \\
\text { anos) }\end{array}$} & \multicolumn{10}{|c|}{ Clínicas } \\
\hline & \multicolumn{2}{|c|}{$\begin{array}{l}\text { Cirurgia } \\
\text { Infantil }\end{array}$} & \multicolumn{2}{|c|}{ Hematologia } & \multicolumn{2}{|c|}{ Ortopedia } & \multicolumn{2}{|c|}{ Outras } & \multicolumn{2}{|c|}{ Total } \\
\hline & $\mathrm{n}$ & $\%$ & $\mathrm{n}$ & $\%$ & $n$ & $\%$ & $\mathrm{n}$ & $\%$ & $\mathbf{N}$ & $\%$ \\
\hline$<1$ & 223 & 9,5 & - & - & 12 & 0,5 & 75 & 3,1 & 310 & 13,1 \\
\hline 1 a 3 & 368 & 15,6 & 86 & 3,6 & 59 & 2,5 & 195 & 8,3 & 708 & 30,0 \\
\hline 4 a 6 & 179 & 7,6 & 72 & 3,0 & 80 & 3,4 & 47 & 2,0 & 378 & 16,0 \\
\hline 7 a 9 & 302 & 12,8 & 91 & 3,9 & 94 & 4,0 & 66 & 2,8 & 553 & 23,5 \\
\hline$\geq 10$ & 216 & 9,1 & 38 & 1,6 & 103 & 4,4 & 54 & 2,3 & 411 & 17,4 \\
\hline Total & 1288 & 54,6 & 287 & 12,1 & 348 & 14,8 & 437 & 18,5 & 2360 & 100,0 \\
\hline
\end{tabular}

Fonte: Do autor.

Do total de procedimentos cirúrgicos identificados, $6,3 \%$ era de pequeno porte, o que inclui exames que necessitaram de suporte anestésico e sala cirúrgica.

Quanto ao tipo de procedimento mais frequente por clínica, destacaram-se na cirurgia infantil apendicectomia $(6,7 \%)$, herniorrafia inguinal $(3,0 \%)$ e laparotomia exploradora $(2,3 \%)$. Na ortopedia prevaleceram correção cruenta de fraturas $(3,7 \%)$, retirada de meios de fixação $(1,6 \%)$ e tenorrafias $(1,2 \%)$. Já na hematologia foram punção liquórica $(3,8 \%)$, quimioterapia intratecal $(3,2 \%)$ e punção de medula óssea (1,8\%) (Tabela 2).

Tabela 2 - Procedimentos cirúrgicos por clínicas entre crianças internadas na unidade pediátrica de um hospital escola público, 2007-2009, Londrina (PR).

\begin{tabular}{|c||c|r|r|}
\hline Clínica & Procedimento Cirúrgico & \multicolumn{1}{c|}{$\mathrm{n}$} & \multicolumn{1}{c|}{$\%$} \\
\hline \hline Cirurgia Infantil & Apendicectomia & 159 & 6,7 \\
\hline Hematologia & Punção liquórica & 89 & 3,8 \\
\hline Ortopedia & Correção de fraturas & 88 & 3,7 \\
\hline Hematologia & Quimioterapia intratecal & 76 & 3,2 \\
\hline Cirurgia Infantil & Herniorrafia inguinal & 71 & 3,0 \\
\hline Cirurgia Infantil & Laparotomia exploradora & 55 & 2,3 \\
\hline Hematologia & Punção de medula óssea & 43 & 1,8 \\
\hline Ortopedia & Retirada de meios de fixação & 38 & 1,6 \\
\hline Ortopedia & Tenorrafias & 28 & 1,2 \\
\hline Outras & Outras & 1713 & 72,7 \\
\hline \hline Total & & 2360 & 100,0 \\
\hline
\end{tabular}

Fonte: Do autor.

Vale ressaltar que as apendicectomias ocorreram mais frequentemente entre o sexo masculino e na faixa etária de sete a nove anos (36,5\%).
Do total de procedimentos cirúrgicos realizados, $379(16 \%)$ foram por causas externas, dos quais $66,5 \%$ eram entre meninos 
(Tabela 3), também na faixa etária de sete a por causas externas para realização de nove anos. Desses, destacaram-se curativo de procedimentos cirúrgicos foi maior nos meses de queimaduras, correções cruentas de fraturas e janeiro, fevereiro, julho e dezembro, totalizando suturas de ferimentos. A frequência de internações 143 casos.

Tabela 3 - Procedimentos cirúrgicos por causas externas e sexo das crianças internadas na unidade pediátrica de um hospital escola público, 2007-2009, Londrina (PR).

\begin{tabular}{|c|c|c|c|r||r|r||c|c|}
\hline \multirow{2}{*}{ Sexo } & \multicolumn{9}{|c|}{ Ano } \\
\cline { 2 - 10 } & \multicolumn{2}{|c|}{2007} & \multicolumn{2}{|c|}{2008} & \multicolumn{2}{|c|}{2009} & \multicolumn{2}{c|}{ Total } \\
\cline { 2 - 10 } & $\mathrm{n}$ & $\%$ & $\mathrm{~N}$ & $\%$ & $\mathrm{n}$ & $\%$ & $\mathrm{n}$ & $\%$ \\
\hline \hline Masculino & $\mathbf{5 7}$ & $\mathbf{1 5 , 0}$ & $\mathbf{4 8}$ & $\mathbf{1 2 , 7}$ & $\mathbf{1 4 7}$ & $\mathbf{3 8 , 8}$ & $\mathbf{2 5 2}$ & $\mathbf{6 6 , 5}$ \\
\hline \hline Feminino & $\mathbf{3 7}$ & $\mathbf{9 , 8}$ & $\mathbf{2 6}$ & $\mathbf{6 , 8}$ & $\mathbf{6 4}$ & $\mathbf{1 6 , 9}$ & $\mathbf{1 2 7}$ & $\mathbf{3 3 , 5}$ \\
\hline \hline Total & 94 & 24,8 & 74 & 19,5 & 211 & 55,7 & 379 & 100,0 \\
\hline
\end{tabular}

Fonte: Do autor.

As correções de malformações congênitas totalizaram $10,8 \%$, com maior prevalência no sexo masculino $(71,8 \%)$ e na faixa etária de um a três anos. Destacaram-se correção de fenda palatina e palatoplastia (17,3\%), seguida das derivações ventrículo peritoniais $(16,9 \%)$ e correção cirúrgica de hipospádia (16,1\%). Não foram encontradas diferenças significativas com relação à época do ano.

Nos três anos de estudo, verificou-se ocorrência de apenas um óbito durante o procedimento intraoperatório.

\section{Discussão}

No presente estudo foi possível identificar que a maior procedência dos procedimentos cirúrgicos foi do próprio município. Predominou a faixa etária de um a três anos e de sete a nove anos. As clínicas mais frequentemente encontradas foram cirurgia infantil, ortopedia e hematologia.

O hospital universitário é uma instituição de grande porte e referência para realização de procedimentos cirúrgicos específicos, como por exemplo, palatoplastia, neurologia, ortopedia e cirurgia pediátrica geral. Também é considerado centro de atendimento ao trauma, principalmente por possuir Unidade de Terapia Intensiva Pediátrica (UTIP) para os casos mais graves e que demandam alta complexidade, além de possuir várias especialidades médicas. Pela própria localização do hospital, é esperado que as demandas cirúrgicas do município sejam as mais frequentes, no entanto também verificou-se crianças procedentes de outros municípios do Paraná e de outros estados.

Outros estudos apontam que hospitais públicos universitários em outras regiões do país são considerados referência para atendimentos cirúrgicos na especialidade pediátrica, bem como possuem maior infraestrutura para realização de cirurgias de pequeno e grande porte (FRACIOZI et al., 2008; REIS et al., 2008; PIMENTA; CALIL; KREBS, 2010; SILVEIRA et al., 2011).

Quanto à oferta de serviços públicos especializados, pesquisa realizada por Dubeux e Carvalho (2009) no Estado de Pernambuco evidenciou que a assistência hospitalar não estava sendo executada conforme o preconizado pelo Modelo Estadual de Regionalização da Assistência à Saúde. Na atenção às urgências/ emergências havia predomínio de casos clínicos/ cirúrgicos de baixa complexidade, como atendimento aos ferimentos leves e cirurgias de apendicectomias, superlotando hospitais e diminuindo possibilidades para assistência de maior complexidade. Os autores concluíram que as limitações na estrutura e no processo de trabalho comprometeram a ofertados hospitais regionais, limitando a assistência hospitalar. 
Entre os atendimentos realizados pela clínica cirúrgica na presente pesquisa, destacou-se a apendicectomia, semelhante ao estudo de Dubeux e Carvalho (2009). A apendicite aguda é considerada uma emergência pediátrica e constitui-se a principal causa de abdome agudo na criança, com maior prevalência no sexo masculino e na faixa etária de quatro a 15 anos (RIBEIRO et al., 2011). Reis et al. (2008) consideram esta enfermidade de resolução cirúrgica mais frequente em crianças e adolescentes. Outros estudos também a reportam como uma das principais causas de hospitalização nesta população (SILVEIRA et al., 2011).

Schweitzer et al. (2010) ressaltam que as complicações precoces da apendicite aguda podem ser infecciosas, como formação de abscesso pélvico ou intra-abdominal, ou tardias, como obstrução intestinal, apesar raras. Além dessas complicações, pesquisa com 341 menores de 16 anos de idade no período de 2004 a 2006, realizado no Serviço de Cirurgia Geral do Hospital do Divino Espírito Santo, Ponta DelgadoPortugal, avaliou prevalência e intensidade da dor aguda pós-operatória, evidenciando que foi superior nas cirurgias de urgência, e ocorreu em 90,9\% dos casos em que houve complicações do procedimento cirúrgico, resultando em maior tempo de internação (3,68 vezes mais). As apendicectomias e laparotomias foram os procedimentos que mais apresentaram episódios dolorosos no pós-operatório (AMARAL et al., 2011).

O segundo procedimento mais frequente observado na presente pesquisa foi punção liquórica pela clínica da hematologia. A incidência de câncer na infância tem aumentado, com estimativa de mais de nove mil casos novos ao ano no Brasil (BRASIL, 2012). Entre esses, merecem destaque as leucemias pela sua alta frequência na população pediátrica, o que demanda terapêutica medicamentosa agressiva com administração de quimioterápicos, sendo alguns casos administrados em ambiente cirúrgico (HAMERSCHLAK, 2012).

Tais procedimentos podem produzir alterações no equilíbrio da criança, porém estas experiências negativas podem ser minimizadas por meio da atenção humanizada e especializada proporcionada pela equipe de enfermagem ao paciente e sua família. Cuidado como este tentam superar uma assistência fragmentada, pautada pelo modelo tecnicista e biomédico. Esse paradigma pode ser modificado durante a formação do profissional da saúde, com desenvolvimento de trabalho em equipe e ações interdependentes e complementares na busca do olhar holístico da criança (THOMAZINE et al., 2008).

As fraturas foram a terceira maior causa cirúrgica neste estudo, representada pela clínica da ortopedia. As fraturas na infância podem ter distintas etiologias dependendo da faixa etária. Traumas contusos em crianças mais novas pode ser resultado de maus tratos e violência, já crianças maiores estão mais expostas a outros tipos de causas externas, como queda, acidente de trânsito, entre outros (CAVALCANTI et al., 2008; FRANCIOSI et al., 2008).

As causas externas ocupam posição de destaque no perfil da morbimortalidade de jovens e crianças entre um e 14 anos de idade, representando a maior causa de morte e ocupando lugar de destaque nas estatísticas de saúde de países desenvolvidos. Além dos custos econômicos e emocionais, os acidentes na infância são responsáveis também por grande parte das mortes e traumatismos não fatais, os quais são causadores de sequelas que repercutem por toda a vida (CAVALCANTI et al., 2008).

Nesteestudo, ascausasexternas forammais frequentes no sexo masculino e na faixa etária de sete a nove anos. As brincadeiras infantis costumam ser diferenciadas por gênero e envolvem fatores socioculturais. Normalmente meninos praticam atividades mais dinâmicas, ficando maior tempo expostos aos riscos de lesões, enquanto meninas costumam ser mais vigiadas e com menor liberdade. Um menino adolescente tem duas vezes mais chances de sofrer um acidente do que uma menina com a mesma idade (BEM et al., 2008). A maior predisposição do sexo masculino aos acidentes e violências também é reportada em outros estudos (GASPAR et al., 2012; MARTINS, 2013). 
Outro achado de destaque corresponde às anomalias congênitas, dentre os principais procedimentos encontrados destaca-se a correção de fenda palatina e palatoplastia, derivações ventrículo peritoniais e correção de hipospadia, concordando com outros autores (POLITA, et al., 2013).

Pesquisa realizada por Biazon e Peniche (2008) para analisar as complicações pós-operatórias em cirurgia primária de lábio e palato de crianças internadas no Hospital de Reabilitação de Anomalias Craniofaciais da Universidade de São Paulo (SP), identificou que a maioria dos pacientes tinha média de idade de 36 meses, com predomínio do sexo masculino. Do total das correções cirúrgicas, 56,6\% foi quiloplastia. A dor foi a complicação mais frequente, seguida da dessaturação de oxigênio e taquicardia. Ressalta-se que a anomalia congênita pode aumentar o risco de morte, principalmente ao associar-se às comorbidades advindas da situação. Ao longo do tempo essas crianças desenvolvem complicações clínicas, o que aumenta o número de internações e gravidade das intercorrências. É necessária uma readequação das políticas de saúde para valorização desse agravo, assim como melhoria nas formas de correção e tratamento, garantindo melhor qualidade de vida às crianças (PIMENTA, et al., 2012).

Segundo Stein (2006), o sucesso da cirurgia depende da aptidão e da habilidade técnica do cirurgião, da indicação cirúrgica, do preparo prévio, do manejo e do cuidado pré-operatório, no qual há dimensionamento dos riscos para prevenir e tratar complicações. Os riscos provindos das cirurgias dependem de fatores próprios do paciente, como estado físico, condições socioeconômicas, hábitos e crenças, tipo de anestesia e procedimento cirúrgico. O tempo de permanência no hospital pode variar de acordo com a influência desses fatores. Além disso, cada especialidade define critérios de alta, os quais devem ser seguidos de forma precisa e rotineira, garantindo menor tempo de internação, redução de custos hospitalares e maior segurança ao paciente (CARVALHO et al., 2010).

\section{Conclusão}

Os resultados mostraram que existem muitos procedimentos no ambiente hospitalar na área pediátrica, variando em clínicas e tipos. Desta forma, para promoção do melhor cuidado às crianças tornase necessário que a equipe multiprofissional conheça esta diversidade, assim como as peculiaridades de cada especialidade. Identificar o perfil das cirurgias realizadas permite aperfeiçoamento de técnicas, adequado preparo pré, trans e pós-operatório, cuidado com a condição física e psicológica da criança e preparo da família para acompanhamento do tratamento e recuperação da criança durante a hospitalização e após a alta. Também ajuda prover materiais e equipamentos para adequando atendimento das demandas, além de favorecer a reorganização dos serviços nos diferentes níveis de atenção à saúde.

Ressalta-se que outros estudos devem ser realizados para analisar o impacto dos procedimentos cirúrgicos pediátricos na vida da criança, família e nas práticas da equipe de saúde, bem como nos custos para o sistema público.

\section{Referências}

AMARAL, L.; MENDES, J.; MARTINS, P.; SILVA, E.; LIMA, M. T. F.; MELO, A. S. Dor aguda na criança operada: a excepção ou a regra? Revista Regional e Terapia da Dor, Cova da Piedade, v. 18, n. 63, p. 50-3, 2011.

BEM, M. A. M.; SILVA, J. L.; SOUZA, J. A.; ARAÚJO, E. J.; PEREIMA, M. L.; QUARESMA, E. $\mathrm{R}$. Epidemiologia dos pequenos traumas em crianças atendidas no Hospital Infantil Joana de Gusmão. $A C M$ : Arquivos Catarinenses de Medicina, Florianópolis, v. 37, n. 2, p. 59-66, 2008.

BIAZON, J.; PENICHE, A. C. G. Estudo retrospectivo das complicações pós-operatórias em cirurgia primária de lábio e palato. Revista da Escola de Enfermagem da USP 2008, 42 (3):519-25.

BRASIL. Ministério da Saúde. Instituto Nacional do Câncer - INCA. Particularidades do câncer infantil. Disponível em: $<\mathrm{http} / /$ www.inca.gov.br/conteudo view.asp?id=343>. Acesso em: 14 maio 2012. 
BROERING, C. V.; CREPALDI, M. A. Preparação psicológica e o estresse de crianças submetidas a cirurgias. Psicologia em Estudo, Maringá, v. 16, n. 1, p. 15-23, 2011.

BROERING, C. V.; CREPALDI, M. A. Preparação psicológica para a cirurgia em pediatria: Importância, técnicas e limitações. Paidéia, Ribeirão Preto, v. 18, n. 39 , p. $61-72,2008$.

CORONEL, M. K. Cirurgia pediátrica: benefícios do acompanhamento psicológico. Perspectiva, Erechim, v. 35, n. 132, p. 81-92, dez. 2011.

CAVALCANTI, A. L.; MARTINS, V. M.; LUCENA, R. N.; GRANVILLE-GARCIA, A. F.; MENEZES, V. A. Morbidade por causas externas em criancas e adolescentes em Campina Grande, Paraíba. ACM: Arquivos Catarinenses de Medicina, Florianópolis, vol. 37, n. 3, p. 27-33, 2008.

CARVALHO, R. W. F.; PEREIRA, C. U; LAUREANOFILHO J. R.; VASCONCELOS, B. C. E. O paciente cirúrgico, Parte I. Revista de Cirurgia e Traumatologia Buco-maxilo-facial, Camaragibe, v. 10, n. 4, p. 85-92, out./dez. 2010.

DUBEUX, L. S.; CARVALHO, E. F. Caracterização da oferta de serviços especializados em hospitais de referência regional: uma contribuição para a regionalização da assistência à saúde no SUS. Revista Brasileira de Saúde Materno Infantil, Recife, v. 9, n. 4, p. 467-76, out./dez. 2009.

FRANCIOZI, C. E. S.; TAMAOKI, M. J. S.; ARAUJO, E. F. A.; DOBASHI, E. T.; UTUMI, C. E.; PINTO, J. A. Politrauma na infância e adolescência: epidemiologia, tratamento e aspectos econômicos em um hospital público. Acta Ortopédica Brasileira, São Paulo, v. 16, n. 5, p. 261-65, 2008.

GASPAR, V. L. V.; SOUZA, E. C. O.; CARMO, J. H.; PEREIRA, W. D. Características de crianças e adolescentes hospitalizados em decorrência de causas externas. $R M M G$ : Revista Médica de Minas Gerais, Belo Horizonte, v. 22, n. 13, p. 287-95, 2012.

HAMERSCHLAK, N. As leucemias no Brasil. Revista Onco\&, São Paulo, n. 14, n. 3, p. 20-4. nov./dez. 2012.

MARTINS, C. B. G. Acidentes e violências na infância e adolescência: fatores de risco e de proteção. Revista Brasileira de Enfermagem, Brasília, v. 66, n. 4, p. 57884, ago. 2013.

PIMENTA, M. S.; CALIL, V. M. L. T.; KREBS, V. L. J. Perfil das malformações congênitas no berçário anexo à maternidade do Hospital das Clínicas da Universidade de São Paulo. Revista de Medicina de São Paulo, São Paulo, v. 89, n. 1, p. 50-56, jan/mar. 2010.
POLITA, N. B.; FERRARI, R. A. P.; MORAES, P. S.; SANT'ANNA, F. L.; TACLA, M. T. G. M. Anomalias congênitas: internações em unidade pediátrica. Revista Paulista de Pediatria, São Paulo, v. 31, n. 2, p. 205-210, 2013.

QUIRINO, D. D.; COLLET, N.; NEVES, A. F. G. B. Hospitalização infantil: concepções da enfermagem acerca da mãe acompanhante. Revista Gaúcha de Enfermagem, Porto Alegre, v. 31, n. 2, p. 300-306, 2010.

REIS, J. M.; OLIVEIRA, D. C. N.; LUCATTO, T. M.; REIS, W. B. Diagnóstico e tratamento de 300 casos de apendicite aguda em crianças e adolescentes atendidos em um hospital universitário. RMMG: Revista Médica de Minas Gerais, Belo Horizonte, v. 18, n. 1, p. 11-15, 2008.

RIBEIRO, S. B.; SILVA, V. M.; GUERRA, E. M. D.; LIMA, M. D. A. Diagnósticos de enfermagem de crianças em pós-operatório de apendicectomia. REME: Revista Mineira de Enfermagem, Belo Horizonte, v. 15, n. 1, p. $19-24,2011$

SCHWEITZER, L. C.; ARAÚUJO, E. J.; SOUZA, J. A.; SGROTT, I.; STAHLSCHMIDT, C.; BATTIST, R. Apendicite aguda complicada na criança: antibioticoterapia em doses múltiplas versus dose única diária. ACM: Arquivos Catarinenses de Medicina, Florianópolis, v. 39, n. 1, p. 57-61, 2010.

SILVEIRA, A.; NEVES, E. T.; FAMOSO, A. F.; DONADUZZI, J. C.; JUNGES, C. F.; ZAMBERLAN, K. C. Caracterização de crianças em tratamento cirúrgico em um hospital escola no Sul do Brasil. Revista de Enfermagem da UFSM, Santa Maria, v. 1, n. 2, p. 174-82, 2011.

SOARES, M. F.; LEVENTHAL, L. C. A Relação entre a equipe de enfermagem e o acompanhante da criança hospitalizada: facilidades e dificuldades. Ciência, Cuidado e Saúde, Maringá, v. 7, n. 3, p. 327-32, 2008.

STEIN, A. T. Recomendações baseadas em evidências. Avaliação pré-operatória e cuidados em cirurgia eletiva. Porto Alegre: Unimed, 2006.

THOMAZINE, A. M.; PASSOS, R. S.; BAY-JÚNIOR, O. G.; COLLET, N.; OLIVEIRA, B. R. G. Assistência de Enfermagem à criança hospitalizada: um resgate histórico. Ciência, Cuidado e Saúde, Maringá, v. 7, supl. 1, p. 145-52, 2008. 